\title{
7
}

\section{SWMM Calibration using Genetic Algorithms}

Benny Wan and William James

The Storm Water Management Model (SWMM) is widely-used to evaluate, analyze and manage problems in both hydraulics and hydrology. In order to improve the reliability of the model, a parameter-optimization approach is required to determine the "best" input parameter sets. Within SWMM, the hydrology module RUNOFF is the best candidate module for uncertainty reduction by parameter optimization.

In this chapter we describe how the genetic algorithm (GA) method was developed to optimize SWMM RUNOFF parameters. The calibration method and its accuracy, efficiency, robustness and reliability are demonstrated. The basic principle of the GA is the same principle that controls the genetic reproduction process with crossover and mutation as the major operations. By applying the genetic algorithm to SWMM with the aid of the sensitivity wizard in the graphical decision support system PCSWMM, a sensitivity-based method for automating the calibration of runoff model was developed.

Overall, the average accuracy of the calibrated model was within $97 \%$ of the target dataset (TD) after approximately 58 cycles of GA calibration program, on the average.

Wan, B. and W. James. 2002. "SWMM Calibration using Genetic Algorithms." Journal of Water Management Modeling R208-07. doi: 10.14796/JWMM.R208-07.

(C) CHI 2002 www.chijournal.org ISSN: 2292-6062 (Formerly in Best modeling practices for Urban Water Systems. ISBN: 0-9683681-6-6) 


\subsection{Introduction}

\subsubsection{SWMM Runoff Models}

The USEPA Storm Water Management Model (SWMM) is one of the most widely-used conceptual-urban-runoff (CUR) models. It is used for estimating flow and water quality variables. SWMM consists of four computational modules: RUNOFF, TRANSPORT, EXTRAN and STORAGE. RUNOFF is used to estimate surface runoff from pervious and impervious areas, and its processes depend on parameters that are uncertain and difficult to estimate. Of the four modules, RUNOFF is the best candidate for improvements in calibration procedures. A poorly-calibrated model might lead to poor designs resulting in four serious impacts: flooding, stream erosion, water quality violations and habitat destruction. In reality, no model represents urban runoff "perfectly" in any watershed. Model errors are often associated with incorrect measurement of model parameters, poor depiction of watershed behavior and structural misrepresentation of the watersheds (Nix, 1994). To overcome these problems, a routine and robust method for adjusting model parameters is required.

\subsubsection{Automatic Calibration}

Traditional calibration methods are applied to create model results that agree closely with measured outputs; agreement is achieved by appropriate choice of model parameters. Parameter selection based on a trial and error approach is often inaccurate and results obtained are time-consuming and unreliable. In order to improve the accuracy of the calibration and the reliability of the models, as well as speed up the calibration process, an automatic calibration method is desirable. Over the last several decades, many different calibration methods, optimization strategies and algorithms have been developed (Kuch, 1994). In this study, with the aid of sensitivity and error analysis, a sensitivity-based automatic calibration for calibrating SWMM RUNOFF is presented.

\subsubsection{Sensitivity-Based GA Calibration Method}

The sensitivity-based GA calibration method consists of four components: (i) sensitivity analysis, (ii) calibration, (iii) determination of the limit or range of the calibration parameters and (iv) performance evaluation of the calibrated model. The GA was selected as the calibration strategy for this study as a result of a literature survey. It is an optimization method based on the hypothesis of 
biological evolution, and thus is part of evolutionary computing. It has a wide range of application including cost and traveling route optimization as well as model calibration.

\subsubsection{Objectives}

An important step in properly using a CUR model is to determine the best set of parameters by calibration (Yan, 1990). Calibration determines model parameters that produce results as close to the observed data as possible. However, discrepancy always occurs between the observed data and model output due to, among other reasons, inaccurate measurement and imperfect parameterization. The discrepancy is considered to be the model uncertainty. To distinguish the imperfect parameterization from uncertainty of the imperfect model structure, an error-free target dataset (TD) was developed to represent the observed data. Errors occur in various forms: time to peak, runoff volume and peak flows. In order to determine the best set of parameters, it is necessary to minimize the errors. When comparing models of varying complexity, for example, it is frequently necessary to have a standard and preferably automatic method of parameter optimization (Siqueira, 2001).

The objectives of this study were to:

1. identify characteristics of the calibration parameters in the input data file from sensitivity analysis and published literature,

2. evaluate the genetic algorithm as a calibration strategy for CUR models,

3. evaluate the applicability and performance of the proposed sensitivity-based calibration method under different conditions, and

4. develop an automatic calibration program in a suitable programming language, such as MS Visual Basic.

\subsubsection{General Procedure}

PCSWMM was selected for the major modeling tool in this study because it has powerful sensitivity-analysis features and is user-friendly. In order to fulfill the first study objective, the sensitivity analysis wizard provided by PCSWMM was used to evaluate all parameters in the input data file.

Generally, the new methodology developed in this study embraces the strategy of automatically determining optimal parameter values. The methodology was evaluated by determining from a deliberately corrupted input data file, an acceptable approximation to the original true input data file, i.e. one that 
reasonably replicates the target output, the original computed response. In other words, the "best" computed response function is assumed to have been produced by the optimal input datafile. The methodology proceeded by first creating several simple "true" input data files and "true" output response function files. The input data file was then corrupted. A suitable objective function, a suitable evaluation function and a method of applying the GA to calibration of a SWMM model, were determined and developed early in the task. The randomly-corrupted input data files were then calibrated using a form of the GA. The performance of this GA methodology was analyzed by considering the importance of the parameters and other user judgments based on experience with the SWMM program.

Parameters were calibrated using a single objective function ("peak runoff rate" was selected for this study) and the performance of the calibration parameters was evaluated by a single evaluation function (the "simple least squares approximation" was selected). The methodology was tested against further, different hydrological conditions.

Finally, the complete sensitivity-based calibration method scheme was implemented in Microsoft Visual Basic. It is shown that the sensitivity-based GA method efficiently and effectively determines a best parameter set (as defined above). During the course of this study, several steps were developed to execute single-event, SWMM-RUNOFF models.

\subsection{The Genetic Algorithm}

\subsubsection{Target Dataset for Conceptual Urban Runoff Models}

Conceptual urban runoff models comprise many mathematical formulations and relationships among various input, state and output variables and parameters (Yan, 1990). Urban runoff models are widely used, for instance in the design of urban stormwater management systems, in pollution control planning, and so on. Because of the complex behavior and characteristics of urban watersheds, and the inherent uncertainty in the applicability of model results to a specific situation, a validation process is required. In the validation process, data must be collected for a wide range of conditions and the model adjusted so that it adequately replicates the observed watershed data. This validation process includes calibration. Calibration refers to a process by which model parameters are adjusted so that one set of system data agrees with another set of independent data (Nix, 1994). In this study, the independent data is the TD. In most real life situations, the independent data refers to measured output or observed data. 
Theoretically, the TD is a set of data that represents the "error-free" or "true" behavior of the urban watershed. Determination of the TD is difficult in urban runoff modeling due to uncertain watershed parameters. An appropriate TD should be carefully selected in order to properly calibrate the models.

\subsubsection{Input, Response, Objective and Evaluation Functions}

In SWMM modeling, four important component functions have to be recognized: the Input Function, Response Function, Objective Function and Evaluation Function. The input function (IF) refers to the driving input of hydrometeorological time series for a CUR, for example rainfall. The output may be the properties of water quantity and water quality, and these are termed the response functions (RFs), for example the flow hydrograph. The objective function $(\mathrm{OF})$ is a statistic or a representative number derived from the response function, chosen to dovetail with the design objectives (James, 2000), for example the peak flow rate. Finally, evaluation functions (EFs) are used to evaluate the performance of the model, which is the comparison between the computed objective functions (COFs) and observed objective functions (OOFs). An example is the sum of the squares of the deviations. In most scientific literature, the term "objective function" usually denotes what we term the "evaluation function". However, the above definitions are used in this study, if only to distinguish between the $\mathrm{OF}$ and the $\mathrm{EF}$.

Multiple OFs are often used in multi-purpose hydrologic models. The most typical objectives for multi-purpose hydrologic models are time of peak, runoff volume and peak flows. In urban runoff models, an important objective and concern is the peak runoff flow rate, and it was selected in this study.

\subsubsection{Sensitivity-Based Calibration Method}

A sensitivity-based system for automating the calibration of the runoff model was developed by Liong et al., (1991) for a watershed in Singapore. They named their calibration method the "knowledge-based calibration system". It comprised three key components:

1. a sensitivity study of calibration parameters;

2. a strategy for parameter selection and assigning values to achieve a match between simulated and measured hydrographs; and

3. knowledge representation scheme used to implement the calibration.

In this present study, the sensitivity-based calibration procedure is similar to the general framework of the knowledge-based calibration system, being based on the synthesis of four components: (i) analyzing for sensitivity, 
(ii) setting calibration parameter limits, (iii) following a calibration strategy and

(iv) conducting an error analysis.

\subsubsection{Robustness and Efficiency}

According to Thyer et al., (1999) robustness is interpreted as the probability of finding the same global optimum (or set of parameters) from a series of independent trials. Efficiency is determined by the number of function evaluations (model runs per optimization trial) required by the algorithm to satisfy prescribed convergence criteria. In order to verify whether the calibration algorithm may be considered to be fair or good, the robustness and efficiency should be identified during the analysis.

\subsubsection{Sensitivity Analysis}

Sensitivity analysis $(\mathrm{SA})$ is performed in order to identify the critical parameters that affect the performance of the model. In general SA consists of four different operations:

1. Alter each parameter in the model one at a time and determine the response.

2. Plot the change in computed output against the changed parameter; yielding the sensitivity gradient.

3. Rank the sensitivity gradients (for each parameter) from highest to zero.

4. Make inferences about the model uncertainty (James, 2000).

SA provides a simple method for both parameter optimization (calibration) and error analysis (James, 2000). The most sensitive parameters are the first to be calibrated.

\subsubsection{Limiting Values of Parameters}

For the same output response, there is an infinite possible combination of parameter sets for the input data. Hence, errors in determining best parameter sets are potentially enormous. In order to confine these errors, determination of limiting values of calibration parameters is critical. The calibration method should not allow parameters to exceed the maximum limit of physically meaningful values. Careful determination of the limits of each calibration parameter increases the accuracy and efficiency of the calibration process. Liong et al. (1995) limit their calibration parameters to $\pm 50 \%$ of the default value, which, as a blanket value for every calibration parameter, seems to the present writers to be arbitrary and unnecessarily high. 


\subsubsection{Genetic Algorithm}

The GA is part of evolutionary computing. In the 1960s, Rechenberg introduced the idea of evolutionary computing in his work "Evolution Strategies". Generally, the evolution-strategy is an optimization method based on the hypothesis of biological evolution. Rechenberg's idea was expanded in the late 1960s and early 1970s. Finally, John Holland and his students and colleagues created the preliminary framework of the GA at the University of Michigan. This lead to Holland's book "Adaption in Natural and Artificial Systems" (1975).

In 1992, GAs were first used in computer programming to perform certain tasks, called "genetic programming" and are still being developed to solve for the optimal value in many applications, such as cost optimization in industry, manufacturing and traveling route determination.

To use a GA, the solution of a problem must be represented as a genome (or chromosome). The GA then creates a population of solutions and applies genetic operators such as mutation and crossover to evolve the solutions in order to find the best one(s).

The GA is based on one main precept: "Good parents have better children", even though this is not true in reality. According to this principle, the process starts with a set of chromosomes (solutions), called a population. Two chromosomes are selected from the population and represent the parents. Each chromosome in the population is evaluated by their degree of fitness, $f(x)$, which is used to decide whether the process should be continued. The second step is to select two chromosomes from a new population as parents and perform a process called crossover. According to the user's assigned crossover probability, children (offspring) might be generated by crossing over the parent's chromosomes. If no crossover is performed, the offspring are an exact copy of their parents. After the process of crossover, mutation takes place. Mutation is a random perturbation process of the genes within the chromosomes according to the mutation probability assigned by the user. After the process of mutation, the new chromosomes are born and are evaluated by their $\mathrm{f}(\mathrm{x})$. In the performance evaluation process, if the $\mathrm{f}(\mathrm{x})$ of the children are better than the parents, this pair of chromosomes is placed into a new population. On the other hand, if the parents are better than the children, the parents' chromosomes are inserted into the next generation. The best $f(x)$ of children or parent will be inserted into the new population for the next iteration. Finally, the whole process is repeated again until the criteria are satisfied. Figure 7.1 describes the process. 


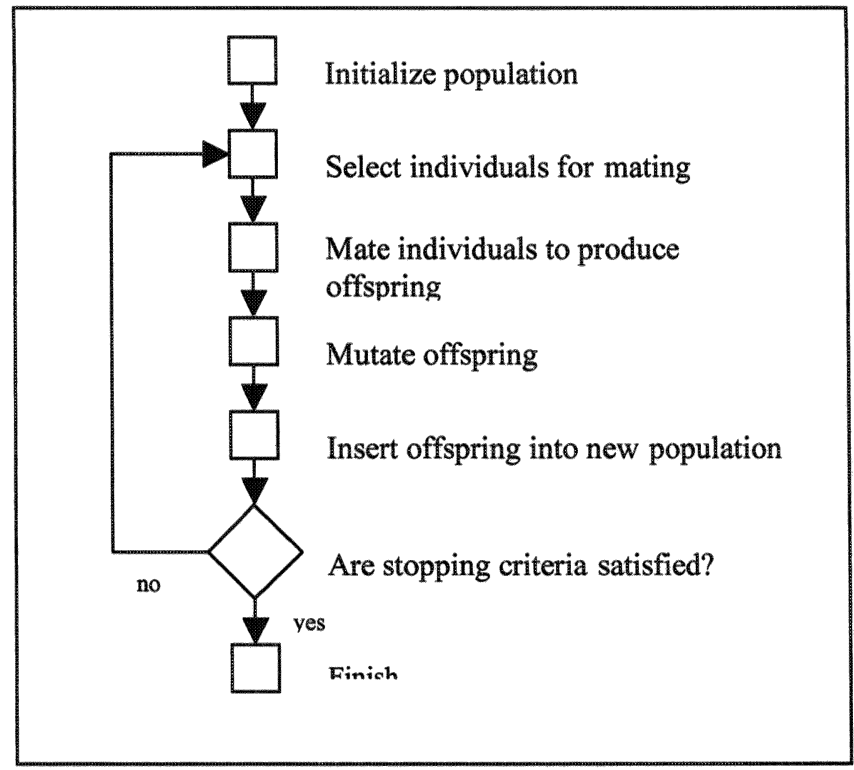

Figure 7.1 GA procedure.

\subsection{Methodology}

\subsubsection{Target Dataset}

While calibration of a model should ideally compare computed output to observed field data, it is impossible to obtain error-free observed data, due to the limitations of the measurement devices and other uncertainties that might occur during the measurement procedure. Hence, in order to verify the consistency and accuracy of this study, a simple SWMM runoff data file was prepared. The TD is the simple runoff output assumed to be error-free. The task of this study is to estimate a "best" parameter set that best matches the TD, or matches it as closely as possible.

\subsubsection{Limitation of Parameters}

For efficient calibration, first estimates of the model parameters should be as close as possible to the true value. Thus a realistic and physically meaningful range of the model parameters of the TD should be identified. A logical rule for determining the limiting values of the parameters is required. More detailed explanation of the determination of parameter limitation is given by Wan (2001). 


\subsubsection{Sensitivity Analysis}

Sensitivity analysis identifies the variables that are most critical to performance of the model. It is also used to optimize their values. In practice, sensitivity analysis changes the model input parameters by a certain percentage (e.g. $\pm 5 \%$ ) and records the change in magnitude of model output. In addition, the impact of various hydrological conditions on the TD can be analyzed by performing the sensitivity analysis on different rainfall events (small, medium and high rainfall) for different durations (short, intermediate and long duration). In this study, the sensitivity analysis is performed using PCSWMM-GIS.

\subsubsection{Genetic Algorithms}

Two runoff data input files represent the parents of the chromosome. After completing the crossover operation, several children are generated and some parameters within the children are mutated randomly. The performance of these children is evaluated by their degree of fitness $(f(x))$, e.g. the error analysis between the computed and TD. Any two of the children or parents may be selected to populate the next generation if their performance is the best among these children and parents. GA calibration is sequential. The process is stopped when an acceptable performance of any member of the population is achieved.

Two problem sets are required to populate the next generation by creating different solution sets (children). In SWMM modeling, two runoff data files (parents) are required initially. The first data file is the "best estimate" input file that was initially built by the modeler and it was for the purposes of this study manually corrupted. The second data file is automatically generated by randomly corrupting the manually-corrupted data file. This random corruption procedure is only applied to sensitive parameters. Generally the TD will be the observed field data. However, as described in an earlier section, the TD in this study is in fact computed ("best estimate" input file). The TD is corrupted (or modified) twice in order to generate the TD's input files (TD-IF); these two corrupted SWMM runoff data files represent the parents.

\section{Crossover Operation}

In microbiological terminology, crossover is an exchange of genes between two chromosomes. There are two different types of crossover: single and multi crossover. Figure 7.2 demonstrates this operation.

In Figure 7.2, WW1, WAREA, WW3, WSLOPE and WW5 are the sensitive SWMM runoff parameters that were selected as the calibration parameters in this example. Initially, the calibration parameters are separated into several different strata. Each strata of the parent that is crossed over is 


\begin{tabular}{|c|c|c|c|c|c|}
\hline & Ww1 & WAREA & Ww3 & WSLOPE & WW5 \\
\hline Parent 1 & 95 & 480 & 20 & 0.0545 & 0.0432 \\
\hline Parent 2 & 86 & 484 & 19.8 & 0.041 & 0.0344 \\
\hline Child 1 & 95 & 480 & 19.8 & 0.041 & 0.0344 \\
\hline Child 2 & 86 & 484 & 20 & 0.0545 & 0.0432 \\
\hline
\end{tabular}

Figure 7.2 Single genetic crossover operation.

\begin{tabular}{|c|c|c|c|c|c|}
\hline & WW1 & WAREA & Ww3 & WSLOPE & Ww5 \\
\hline Parent 1 & 95 & 480 & 20 & 0.0545 & 0.0432 \\
\hline Parent 2 & 86 & 484 & 19.8 & 0.041 & 0.0344 \\
\hline Child 1 & 95 & 480 & 19.8 & 0.041 & 0.0432 \\
\hline Child 2 & 86 & 484 & 20 & 0.0545 & 0.0344 \\
\hline
\end{tabular}

Figure 7.3 Multi crossover operation.

determined by the crossover probability. In Figure 7.3, the crossover probability is $40 \%, 40 \%$ and $20 \%$, and, as a result, WW1 and WAREA are in the $1^{\text {st }}$ strata, WW3 and WSLOPE are in the $2^{\text {nd }}$ strata, and WW5 is in the $3^{\text {rd }}$ strata. The number of parameters in each stratum can be determined by the following equation:

\section{Number of parameters in each strata $=$ \\ Crossover Probability $x$ Total Number of Parameters}

According to Figure 7.3, two parents generate two children after the crossover operation. This kind of crossover is the most typical GA procedure. However, it is possible to generate more than two children if multi crossover is used. In reality parents can have many children. The maximum possible combination of crossing over the multiple strata of the parent solution sets is examined in this study.

In Figure 7.4, six possible children are born from both parents with three strata. The total number of children depends on the number of strata and is decided by the user. The total maximum possible number of children is determined by the following equation:

$$
\text { Total Maximum Possible Children }=2^{n}-N_{\text {parent }}
$$




\begin{tabular}{|c|c|c|c|c|c|}
\hline & WW1 & WAREA & Ww3 & WSLOPE & WW5 \\
\hline Parent 1 & 95 & 480 & 20 & 0.0545 & 0.0432 \\
\hline Parent 2 & 86 & 484 & 19.8 & 0.041 & 0.0344 \\
\hline Child 1 & 95 & 480 & 19.8 & 0.041 & 0.0432 \\
\hline Child 2 & 86 & 484 & 20 & 0.0545 & 0.0344 \\
\hline Child 3 & 95 & 480 & 20 & 0.0545 & 0.0344 \\
\hline Child 4 & 86 & 484 & 19.8 & 0.041 & 0.0432 \\
\hline Child 5 & 95 & 480 & 19.8 & 0.041 & 0.0344 \\
\hline Child 6 & 86 & 484 & 20 & 0.0545 & 0.0432 \\
\hline
\end{tabular}

Figure 7.4 Maximum number of children for 2 parents, 3 strata.

where:

$$
\begin{aligned}
n & =\text { total number of strata, and } \\
N_{\text {parent }} & =\text { total number of parents }
\end{aligned}
$$

After the crossover operation, the next procedure is mutation.

\section{Mutation Operation}

Mutation is a part of the genetic reproduction process. The purpose of this operation is to perturb the genetic information within a chromosome. In practice, each parameter that was contained in a solution set of each child will be perturbed by adding or subtracting a small number. Various types of mutation encoding are available - in this study, "value encoding" is employed. The parameter perturbation is decided by the user-defined mutation probability. Figure 7.5 shows the procedure.

In Figure 7.5, the subcatchment area (WAREA) and subcatchment slope (WSLOPE) are chosen to be the parameters to mutate and the mutation values are $+5 \%$ and $-5 \%$, respectively. The user defines this mutation value and the mutated parameters are randomly selected. In this example, the mutation value is fixed at $\pm 5 \%$. However, this value is further investigated in this study in order to improve the calibration process. Finally, the order of mutation is calculated by the following equation:

$$
M_{\text {order }}=(-1)^{n-1}
$$

where:

$$
\begin{aligned}
n & =\text { parameter number, and } \\
M_{\text {order }} & =\text { mutation order }
\end{aligned}
$$




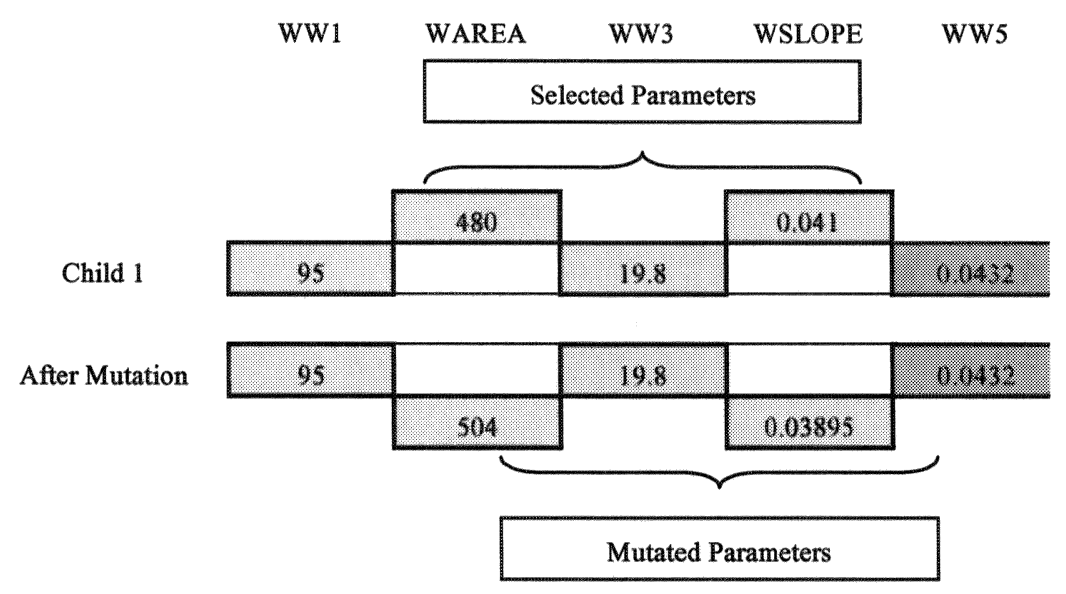

Figure 7.5 Mutation operation.

Once the crossover and mutation operations are completed, the next step is to input the new SWMM runoff parameter sets into PCSWMM and run the model. The objective function (peak runoff flow rate) is determined by PCSWMM.

\subsubsection{Rainfall Events vs. Number of Calibration Parameters}

In a system of simultaneous equations, a unique solution can only be obtained if there are as many distinct equations as there are solutions. It is impossible to obtain a unique solution to a single equation with two variables - an infinite combination of solutions result. An additional equation is required in order to solve the system. In order to calibrate a model with one calibration parameter only, one single rainfall event is needed. However, if two calibration parameters are required, two distinctly different rainfall events are needed. The calibration method is based on the hypothesis: If $n$ calibration parameters are sensitive in the model, $n$ distinct rainfall events are required. The following equations show the preliminary idea of calibrating the model with multiple rainfall events:

$$
\begin{aligned}
& \varepsilon_{1}=\alpha_{11} \cdot \Delta p_{11}+\alpha_{12} \cdot \Delta p_{12}+\ldots \ldots \ldots+\alpha_{1 n} \cdot \Delta p_{1 n} \\
& \varepsilon_{2}=\alpha_{21} \cdot \Delta p_{21}+\alpha_{22} \cdot \Delta p_{22}+\ldots \ldots \ldots+\alpha_{2 n} \cdot \Delta p_{2 n} \\
& \cdot \\
& \cdot \\
& \varepsilon_{n}=\alpha_{n 1} \cdot \Delta p_{n 1}+\alpha_{n 2} \cdot \Delta p_{n 2}+\ldots \ldots \ldots+\alpha_{n n} \cdot \Delta p_{n n}
\end{aligned}
$$


where:

$$
\begin{aligned}
\varepsilon= & \text { error between computed objective function (COF) } \\
& \text { and observed objective function (OOF), } \\
\alpha= & \text { sensitivity gradient, } \\
\Delta p= & \text { change in parameter, and } \\
\text { (subscript: } n= & \text { rainfall event) }
\end{aligned}
$$

\subsubsection{Computation Approach}

In order to test the performance of the GA calibration process, a GA calibration program (GA-CP) was created in Visual Basic. Two TD-IFs were created in the analysis, (i) Simple TD-IF and (ii) Complex TD-IF.

\subsection{Results and Discussion}

\subsubsection{Simple TD-IF}

For the first test of the GA-CP, a simple TD-IF was used. Table 7.1 and

\begin{tabular}{|c|c|c|c|}
\hline Subcatchment ID & 100 & 200 & 300 \\
\hline Width & $30.48 \mathrm{~m}$ & $15.24 \mathrm{~m}$ & $45.72 \mathrm{~m}$ \\
\hline Area & $121.41 \mathrm{ha}$ & 40.47 ha & 161.87 ha \\
\hline$\%$ Imperviousness & $20 \%$ & $30 \%$ & $10 \%$ \\
\hline Slope & 0.001 & 0.001 & 0.002 \\
\hline Impervious Area Manning's Roughness & 0.04 & 0.04 & 0.04 \\
\hline Pervious Area Manning's Roughness & 0.3 & 0.3 & 0.3 \\
\hline Impervious Area Depression Storage & $1.27 \mathrm{~mm}$ & $1.27 \mathrm{~mm}$ & $1.27 \mathrm{~mm}$ \\
\hline Pervious Area Depression Storage & $2.54 \mathrm{~mm}$ & $2.54 \mathrm{~mm}$ & $2.54 \mathrm{~mm}$ \\
\hline \multicolumn{4}{|c|}{ Green-Ampt Infiltration Parameters } \\
\hline Average Capillary Suction & $101.6 \mathrm{~mm}$ & $76.2 \mathrm{~mm}$ & $101.6 \mathrm{~mm}$ \\
\hline Saturated Hydraulic Conductivity & $25.4 \mathrm{~mm} / \mathrm{hr}$ & $25.4 \mathrm{~mm} / \mathrm{hr}$ & $12.7 \mathrm{~mm} / \mathrm{hr}$ \\
\hline Initial Moisture Deficit & 0.34 & 0.34 & 0.34 \\
\hline Maximum Infiltration Volume & $127 \mathrm{~mm}$ & $127 \mathrm{~mm}$ & $127 \mathrm{~mm}$ \\
\hline
\end{tabular}
Table 7.2 list the hydraulic and hydrologic characteristics of the simple TD-IF.

Table 7.1: Hydraulic characteristics of the TD-IF.

\begin{tabular}{lccc}
\hline Channel/Pipes ID & 10 & 20 & 30 \\
\hline Channel/Pipes Type & Circular Pipe & Circular Pipe & Circular Pipe \\
Diameter & $0.9 \mathrm{~m}$ & $0.9 \mathrm{~m}$ & $0.9 \mathrm{~m}$ \\
Length & $1524 \mathrm{~m}$ & $304.8 \mathrm{~m}$ & $152.4 \mathrm{~m}$ \\
Invert Slope & 0.01 & 0.01 & 0.01 \\
Manning's Roughness Coeff. & 0.03 & 0.03 & 0.03
\end{tabular}

Table 7.2 Hydrologic characteristics of the TD-IF. 


\section{Sensitivity Analysis}

The proposed sensitivity-based GA calibration method uses a sensitivity analysis to obtain candidate calibration parameters. The sensitivity analysis for the simple TD-IF is shown in Figure 7.6.

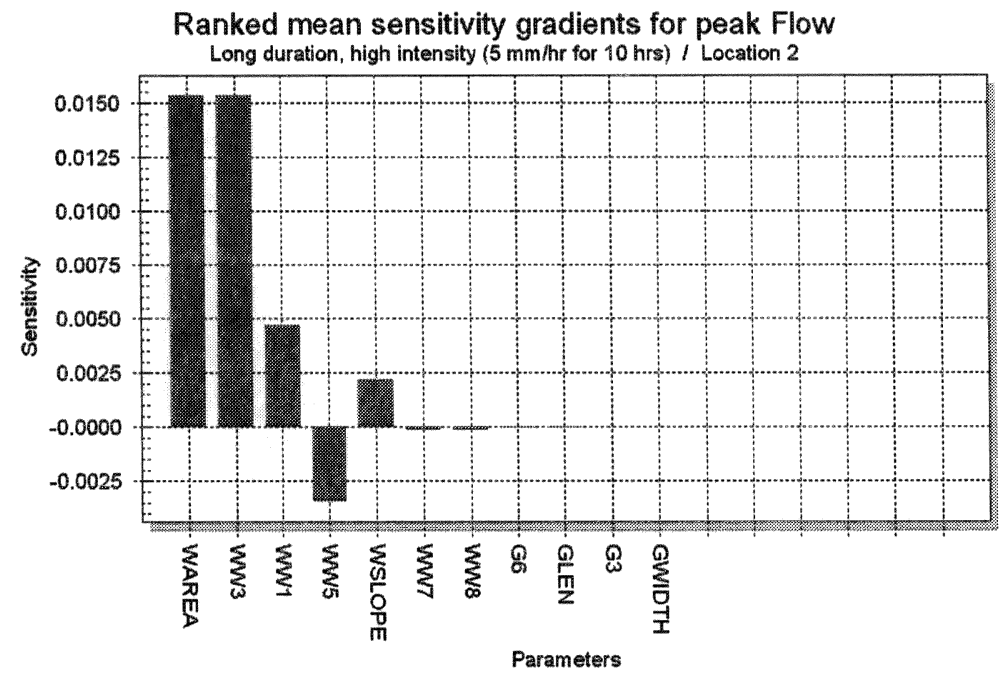

Figure 7.6 Results of sensitivity analysis for simple TD-IF.

According to Figure 7.6, eleven parameters were initially thought to be sensitive. The channel roughness coefficient (G6), length of the channel (GLEN), invert slope of the channel (G3) and width of the channel (GWIDTH) were not considered in the calibration process due to their insignificant sensitivity. As a result, only seven parameters were selected and their descriptions are given in Table 7.3.

Table 7.3 Definition of sensitive parameters.

\begin{tabular}{cl}
\hline SWMM Parameters & \multicolumn{1}{c}{ Description } \\
\hline WW1 & Subcatchment width \\
WAREA & Subcatchment area \\
WSLOPE & Slope of the subcatchment \\
WW3 & Percent Imperviousness \\
WW5 & Manning's n \\
WW7 & Impervious area depression storage \\
WW8 & Pervious area depression storage \\
\hline
\end{tabular}

As mentioned earlier, the calibration process requires that the number of distinct rainfall events be equal to the number of calibration parameters. Since seven calibration parameters were determined in the sensitivity analysis, seven 
distinct rainfall events were selected: $2.5,7.5,13,18,22.5,28$ and $30 \mathrm{~mm} / \mathrm{h}$ for $1 \mathrm{~h}$ precipitation duration. In order to determine the calibration parameters for different hydrological conditions, these rainfall events were used to determine the sensitivity of calibration parameters for different hydrological conditions. Table 7.4 summarizes the results of the sensitivity analysis for different rainfall events.

Table 7.4 Ranking of the sensitive parameters vs. different rainfall.

\begin{tabular}{cccccccc}
\hline $\begin{array}{c}\text { Rainfall } \\
(\mathrm{mm} / \mathrm{hr} \text { ) }\end{array}$ & \multicolumn{3}{c}{ Most sensitive parameter } & $\rightarrow$ & \multicolumn{2}{c}{ Least sensitive parameter } \\
\hline 2.5 & WW5 & WW7 & WW8 & WW1 & WSLOPE & WAREA & WW3 \\
7.5 & WW5 & WW1 & WSLOPE & WW7 & WW8 & WAREA & WW3 \\
13 & WW5 & WW1 & WSLOPE & WW7 & WW8 & WAREA & WW3 \\
18 & WW5 & WW1 & WSLOPE & WAREA & WW3 & WW7 & WW8 \\
22.5 & WW5 & WW1 & WSLOPE & WAREA & WW3 & WW7 & WW8 \\
28 & WW5 & WW1 & WSLOPE & WAREA & WW3 & WW7 & WW8 \\
30 & WW5 & WW1 & WSLOPE & WAREA & WW3 & WW7 & WW8 \\
\hline
\end{tabular}

According to Table 7.4, an agreement for the sensitive parameters was found, and these seven parameters were considered candidates for calibration.

\section{GA-CP for Simple TD-IF}

After approximately 49 cycles of GA-CP, the best parameter set was obtained with the $\mathrm{f}(\mathrm{x})$ (sum of the squares of the deviations) of $4.892218 \mathrm{E}-03$. Figures 7.7 and 7.8 show the calibration plots before and after using the GA program for fve of the seven storms.

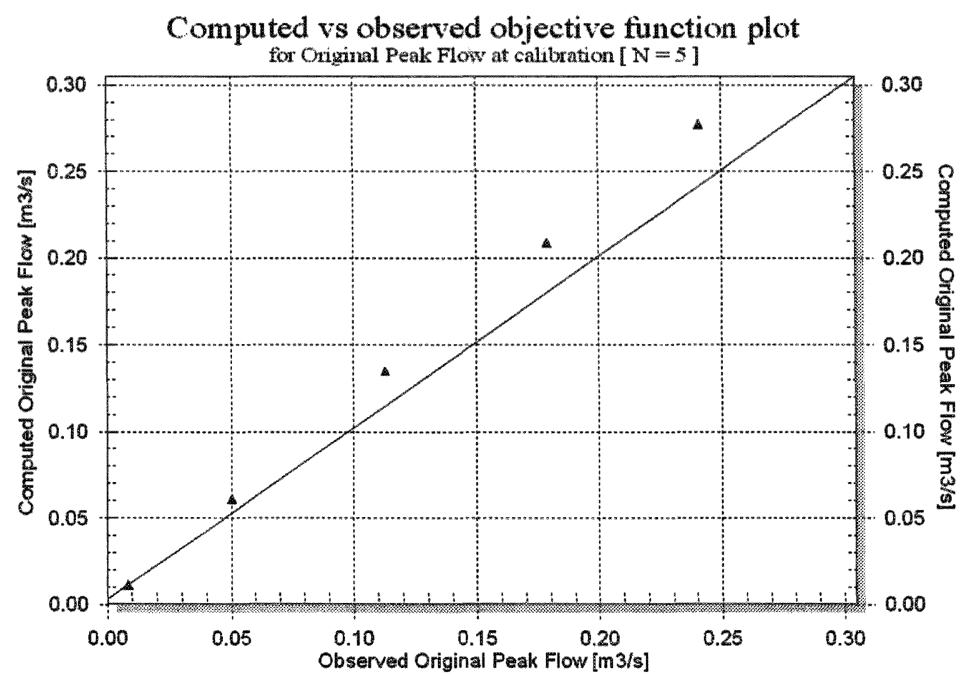

Figure 7.7 Calibration plot of TDIF2.dat (before calibration). 


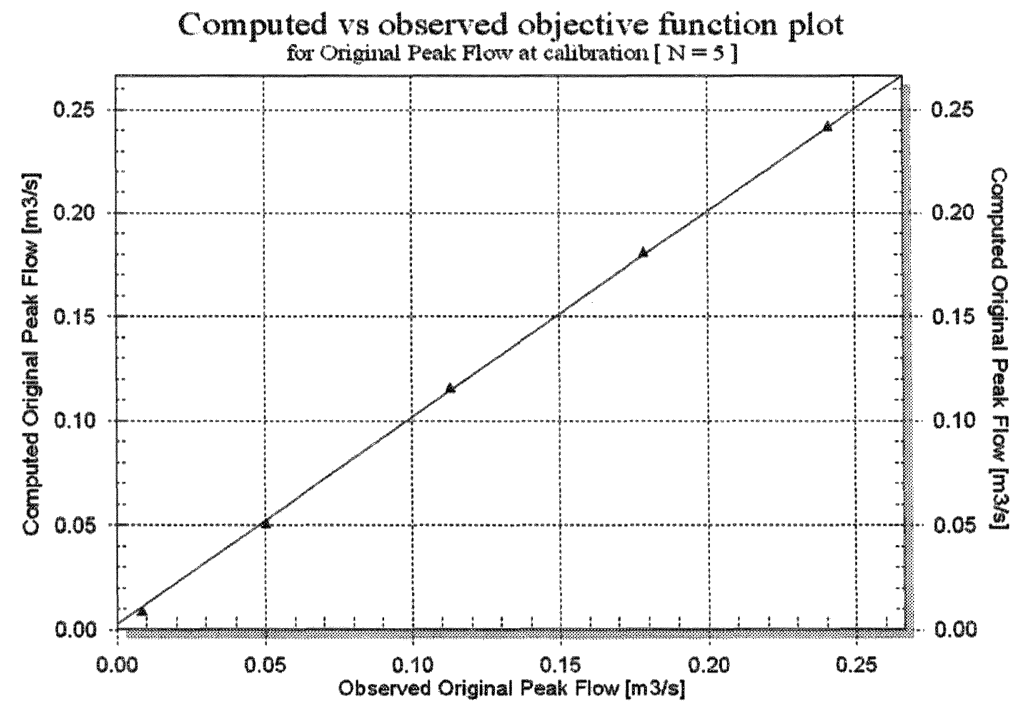

Figure 7.8 Calibration plot of best parameter set (after calibration).

Since five calibration parameters were used, the tolerance of this analysis was 0.0125 . After the first six runs, the $\mathrm{f}(\mathrm{x})$ of $5.99459 \mathrm{E}-03$ was achieved and the tolerance was met. Reaching the tolerance within six runs proved that the sensitivity-based GA calibration method could consistently and efficiently determine the best parameter set.

In order to complete the second task, the GA calibration program continued with the same mutation range of $5 \%$ for another 30 runs and the $f(x)$ of $4.984318 \mathrm{E}-03$ was estimated in the $36^{\text {th }}$ run. The mutation range was changed to $3 \%$ after the $36^{\text {th }}$ run in order to determine a better answer. A lower value of $\mathrm{f}(\mathrm{x}), 4.918529 \mathrm{E}-03$, was achieved in the $44^{\text {th }}$ run. Subsequently, the mutation range of $1 \%$ and $0.5 \%$ was used to determine an even lower $\mathrm{f}(\mathrm{x})$. Finally, $4.892218 \mathrm{E}-03$ was determined in the $49^{\text {th }}$ run. Figure 7.9 shows the highest peak runoff rates. The trend of the computed output for various cycles of GA-CP is shown.

Table 7.5 lists the labels that appear in Figure 7.9 and summarizes the results.

The overall performance of calibration parameters was considered to be acceptable. In fact, the $\mathrm{f}(\mathrm{x})$ of $4.892218 \mathrm{E}-03$ represents a $4.5 \%$ difference between the OOF and COF6. This proves that the GA-CP can reasonably accurately determine suitable parameter sets. 


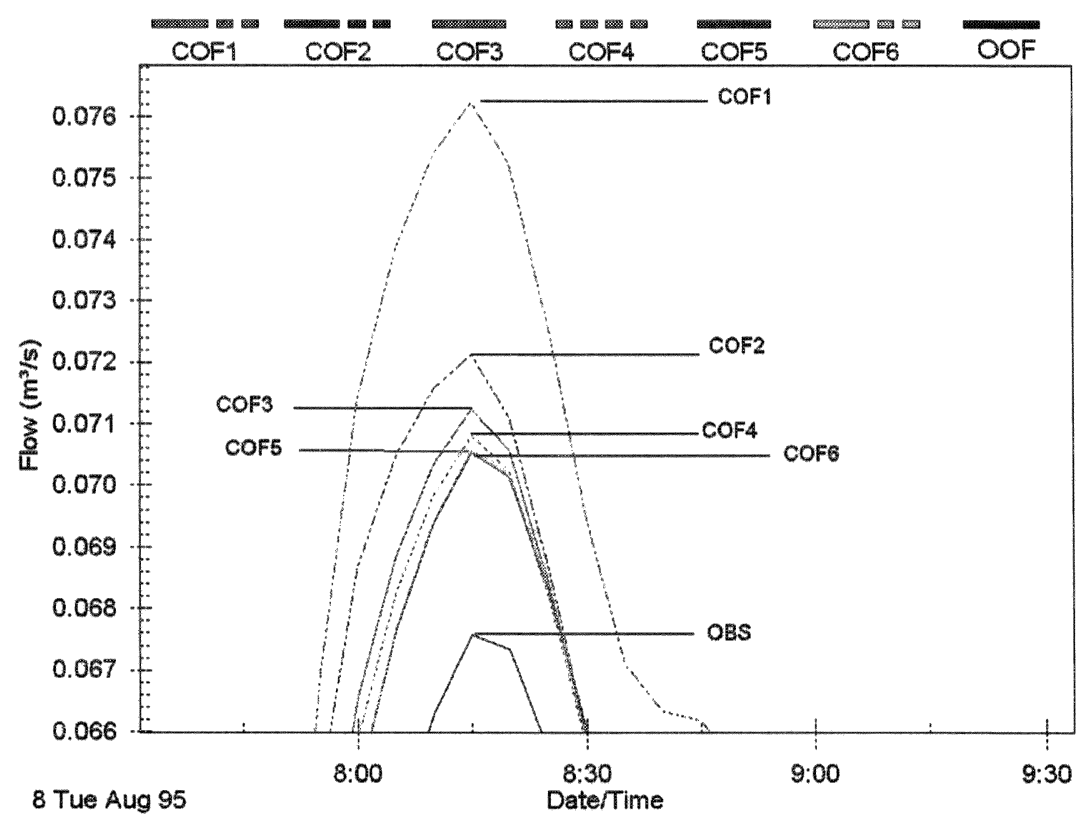

Figure 7.9 Highest peak runoff rate.

Table 7.5 Labels and results of GA-CP for simple TD-IF.

\begin{tabular}{llccc}
\hline Labels & \multicolumn{1}{c}{ Definition } & $\mathrm{f}(\mathrm{x})$ & Max. flow & Cycle \\
\hline OOF & Observed Objective Function & N/A & $0.06756 \mathrm{~m}^{3} / \mathrm{s}$ & N/A \\
COF1 & Computed Objective Function & N/A & $0.07623 \mathrm{~m}^{3} / \mathrm{s}$ & N/A \\
COF2 & $2^{\text {nd }}$ COF & $5.99459 \mathrm{E}-03$ & $0.07211 \mathrm{~m}^{3} / \mathrm{s}$ & 6 \\
COF3 & $3^{\text {rd }}$ COF & $4.984318 \mathrm{E}-03$ & $0.07124 \mathrm{~m}^{3} / \mathrm{s}$ & 36 \\
COF4 & $4^{\text {th }}$ COF & $4.918529 \mathrm{E}-03$ & $0.07081 \mathrm{~m}^{3} / \mathrm{s}$ & 44 \\
COF5 & $5^{\text {th }}$ COF & $4.892218 \mathrm{E}-03$ & $0.07057 \mathrm{~m}^{3} / \mathrm{s}$ & 56 \\
COF6 & $6^{\text {th }}$ COF & $4.892218 \mathrm{E}-03$ & $0.07057 \mathrm{~m}^{3} / \mathrm{s}$ & 61 \\
\hline
\end{tabular}

\subsubsection{Complex TD-IF}

The purpose of this stage is to determine the whether the GA-CP can calibrate a more complex runoff file. A complex TD-IF was created with 16 subcatchments and 19 circular pipes with the identical attributes of both hydrologic and hydraulic parameters. Figure 7.10 shows the connectivity of the complex TD-IF. 


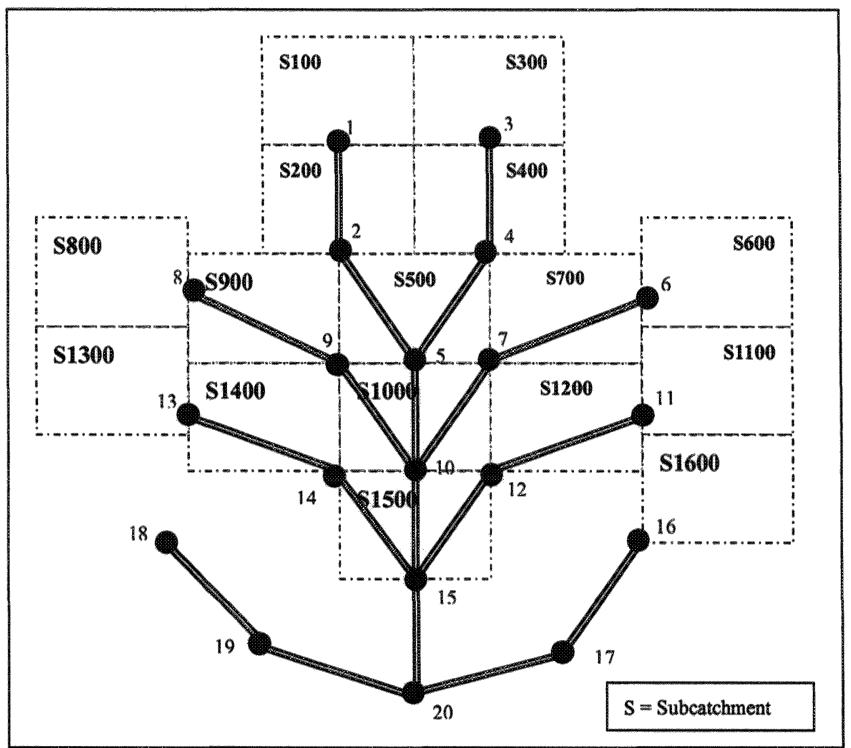

Figure 7.10 Connectivity of complex TD-IF.

Sensitivity analysis was subsequently performed to identify the calibration parameters. Figure 7.11 shows the results of the sensitivity analysis.

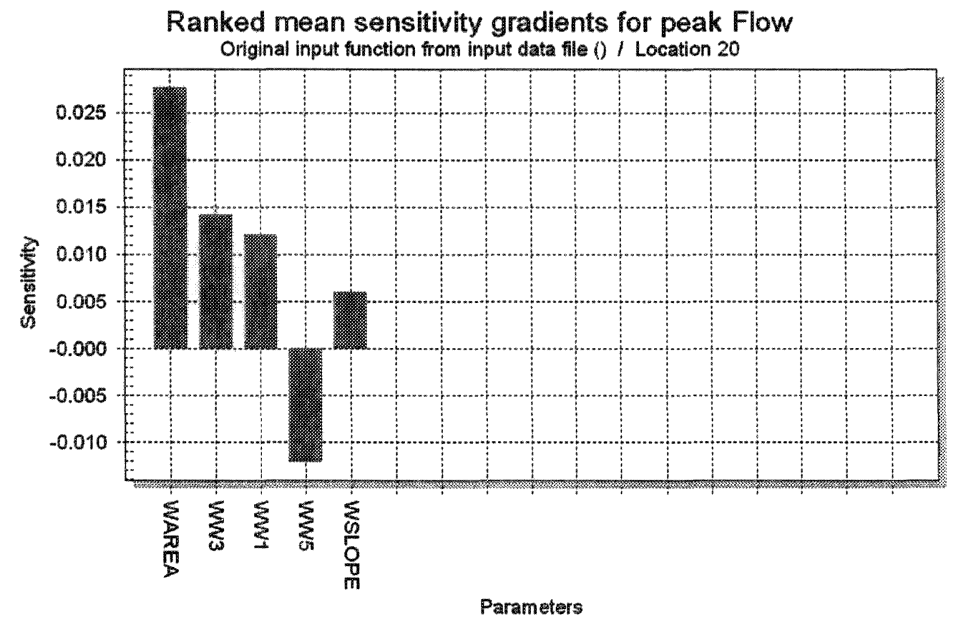

Figure 7.11 Sensitivity gradient of complex TD-IF. 
Again, five rainfall events were used in the calibration process due to the number of sensitive parameters. These rainfall events were $2.5 \mathrm{~mm} / \mathrm{h}$, $7.5 \mathrm{~mm} / \mathrm{h}, 13 \mathrm{~mm} / \mathrm{h}, 18 \mathrm{~mm} / \mathrm{h}$ and $22.5 \mathrm{~mm} / \mathrm{h}$ for $1 \mathrm{~h}$ precipitation duration.

After approximately 50 cycles, an $\mathrm{f}(\mathrm{x})$ of $3.378113 \mathrm{E}-04$ was determined. Calibration plots for Complex TD-IF and best parameter set are shown in Figure 7.12. After calibration the points plot on the line.

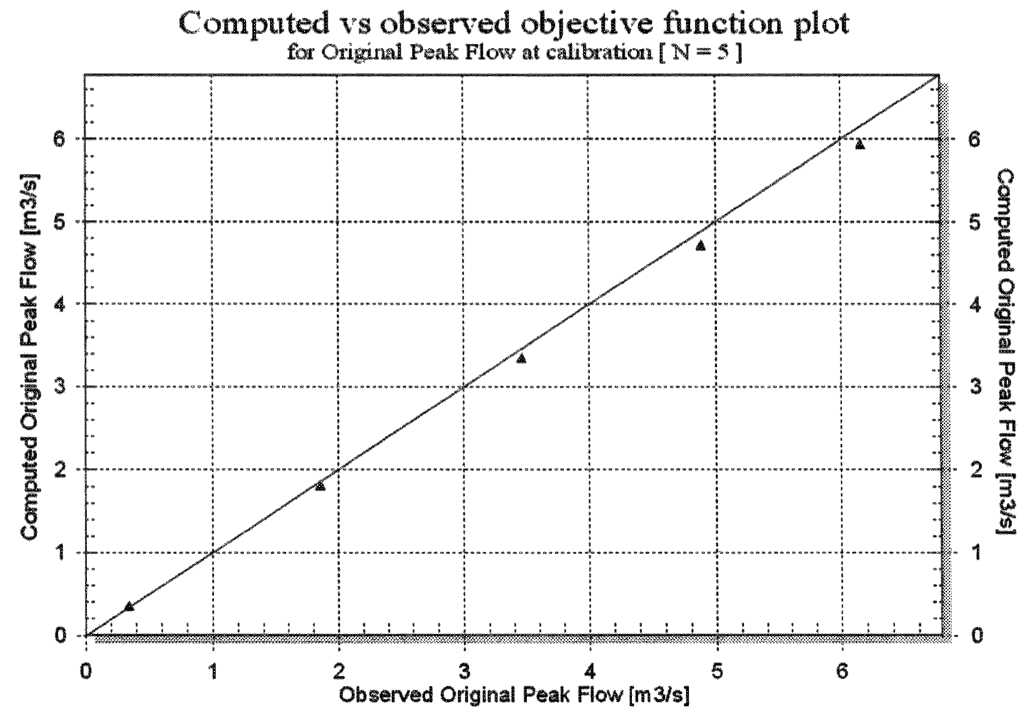

Figure 7.12 Calibration plot of complex TD-IF (before calibration).

In Figure 7.12, the error for each rainfall event even before calibration was very small. This indicates that calibration becomes less important in a complex hydrologic model because the connectivity of the discretized watersheds accounts for much of the hydrograph shape. The consistency and accuracy of GA-CP is shown by the reduction of error even when the start-up errors are not very large, and we submit that the capability of the GA-CP is thus proven for complex systems.

\subsubsection{Summary of Performance of GA-CP}

After performing GA-CP on simple and complex TD, Table 7.6 summarizes the results.

According to Table 7.6, the GA-CP could determine the "best" parameter set within an average of $97 \%$ after 58 cycles of GA-CP in average. The number of cycles that were required to determine the best $\mathrm{COF}$ was not very convincing 
Table 7.6 Summary of performance of GA-CP.

\begin{tabular}{lll} 
& Simple TD-IF & Complex TD-IF \\
\hline OOF & $0.06756 \mathrm{~m}^{3} / \mathrm{s}$ & $2.001 \mathrm{~m}^{3} / \mathrm{s}$ \\
Best COF & $0.07057 \mathrm{~m}^{3} / \mathrm{s}$ & $1.989 \mathrm{~m}^{3} / \mathrm{s}$ \\
Accuracy & $95.5 \%$ & $99.4 \%$ \\
Number of Cycle & 61 & 50 \\
\hline
\end{tabular}

in term of efficiency. However, the $\mathrm{f}(\mathrm{x})$ of the best COF was much lower than the tolerance. Hence, GA-CP is considered to be an efficient, robust, accurate and reliable method for calibrating a SWMM RUNOFF model.

\subsection{Conclusions}

A sensitivity-based genetic algorithm method for calibration of SWMM has been developed and evaluated in this work. The calibration method was developed using a combination of sensitivity analysis and a genetic algorithm optimization. This calibration method produced a set of parameters that converged to their true values.

The characteristics of the proposed calibration method are:

1. comprehensive sensitivity analysis,

2. efficient and accurate estimation of the model parameters,

3. calibration of complex SWMM runoff models, and

4. the robustness of the automatic GA calibration program.

Model parameters were estimated using one statistic of a typical hydrologic response, namely, peak runoff rate. Simple least squares approximation (one dimensional form) was selected to evaluate the performance of the calibration method. Different SWMM runoff input files were used to determine the characteristics of the calibration method and the GA parameters.

Using the first SWMM input file, the preliminary sensitivity-based GA calibration was tested. In addition, the GA parameters were optimized using the first SWMM input file. The consistency and accuracy of determining the best parameter set were verified by using the second SWMM input file. Finally, the last SWMM input file was used to test the proposed GA method for complex SWMM models.

The results indicated that the parameter estimates using the proposed methodology provide excellent overall approximation for the selected objective. Efficiency, robustness, accuracy and reliability of the proposed method appear to be reasonable. 
The results lead to the following conclusions:

1. The theory of genetic algorithm optimization for calibrating the SWMM parameters has been found to be effective.

2. This research has demonstrated that the calibration method can improve the accuracy and reliability of the estimates of the model parameters.

Robustness and efficiency can be evaluated by using the automatic program developed in this study for the genetic algorithm calibration method.

\section{References}

Holland, J. H., (1975), Adaptation in natural and artificial systems : an introductory analysis with applications to biology, control, and artificial intelligence, Ann Arbor, MI, University of Michigan Press. ISBN 0-262-58111-6

James, W. (2000), Rules for Responsible Modeling 2nd Edition, CHI, Guelph, Ontario. ISBN0-9683681-5-8

Kuch, A. J. (1994), A Sensitivity Analysis Shell for Complex Continuous Water Quality Management Modelling. M. Sc. Thesis, University of Guelph. Ca., 120 pp.

Liong, S.Y., Ibrahim, Y. (1995), Catchment Calibration Using Fractional-Factorial and Central-Composite-Designs-Based Response Surface, Journal of Hydraulic Engineering, Vol. 121 (6), p. 507-617.

Liong, S.Y., Chan, W.T., Lum, L.H. (1991), Knowledge-Based System for SWMM Runoff Component Calibration, Journal of Water Resources Planning and Management, Vol. 117 (5), p. 507-524.

Nix, S. J., (1994), Urban Stormwater Modeling and Simulation, CRC Press, Inc., Florida. ISBN0-87371-527-6

Rechenberg, I., (1973), Evolution Strategies, Stuttgart-Bad Cannstatt : FrommannHolzboog, Germany, ISBN 377280374

Siqueira, E., (2001), Model Complexity and Uncertainty, presented to 2001 Stormwater \& Urban Water Systems Modeling Conference, Toronto, organized by CHI, Guelph, Ontario.

Thyer, M., Kuczera, G., and Bates, C. (1999), Probabilistic Optimization for Conceptual Rainfall-Runoff Models: A Comparison of the Shuffled Complex Evolution and Simulated Annealing Algorithms, Water Resources Research, Vol. 35, p. 767-773

Wan, B. (2001), Auto-Calibration of SWMM RUNOFF using Sensitivity-Based Genetic Algorithm, M.Sc. Thesis, University of Guelph, Canada.

Yan, J. (1990), Parameter Estimation for Multipurpose Hydrologic Models, Ph.D. Thesis, Department of Agricultural Engineering, Oklahoma State University, Oklahoma. 